\title{
Enabling Data Analytics and Machine Learning for 5G Services within Disaggregated Multi-Layer Transport Networks
}

\author{
Ramon Casellas, Ricardo Martínez, Luis Velasco ${ }^{+}$, \\ Ricard Vilalta, Pablo Pavón", Daniel King", Raül Muñoz \\ Centre Tecnològic de Telecomunicacions de Catalunya (CTTC/CERCA), Castelldefels, Spain \\ ${ }^{+}$Universitat Politècnica de Catalunya, UPC, Barcelona, Spain \\ * Universidad Politécnica de Cartagena, UPCT, Cartagena, Spain. \\ \# Old Dog Consulting, UK \\ e-mail: ramon.casellas@cttc.es
}

\begin{abstract}
Recent advances, related to the concepts of Artificial Intelligence (AI) and Machine Learning (ML) and with applications across multiple technology domains, have gathered significant attention due, in particular, to the overall performance improvement of such automated systems when compared to methods relying on human operation. Consequently, using $\mathrm{AI} / \mathrm{ML}$ for managing, operating and optimizing transport networks is increasingly seen as a potential opportunity targeting, notably, large and complex environments.

Such AI-assisted automated network operation is expected to facilitate innovation in multiple aspects related to the control and management of future optical networks and is a promising milestone in the evolution towards autonomous networks, where networks self-adjust parameters such as transceiver configuration.

To accomplish this goal, current network control, management and orchestration systems need to enable the application of AI/ML techniques. It is arguable that Software-Defined Networking (SDN) principles, favouring centralized control deployments, featured application programming interfaces and the development of a related application ecosystem are well positioned to facilitate the progressive introduction of such techniques, starting, notably, in allowing efficient and massive monitoring and data collection.

In this paper, we present the control, orchestration and management architecture designed to allow the automatic deployment of 5G services (such as ETSI NFV network services) across metropolitan networks, conceived to interface $5 \mathrm{G}$ access networks with elastic core optical networks at multi Tb/s. This network segment, referred to as Metro-haul, is composed of infrastructure nodes that encompass networking, storage and processing resources, which are in turn interconnected by open and disaggregated optical networks. In particular, we detail subsystems like the Monitoring and Data Analytics or the in-operation planning backend that extend current SDN based network control to account for new use cases.
\end{abstract}

Keywords: Data Analytics, SDN/NFV Control of Optical / Multi-Layer Networks.

\section{INTRODUCTION}

The benefits of SDN based architectures go well beyond the separation of data and control planes, and centralized controllers. The inherent programmability and the extended use of open Application Programming Interfaces (APIs) is enabling innovation in areas related to monitoring, autonomous networking and overall operational feedback loop improvements. In particular, and driven by the ever-increasing amount of devices, and the raise of disaggregated deployments, control, orchestration and management (COM) systems need not only to automate service provisioning and recovery, but need also to monitor network and computing performances.

This goal is not only to react to events such as failures, but also to foresee degradations or capacity exhaustion. Monitoring and telemetry are automated tasks by which measurements data from network and IT devices, applications and services, are collected, transmitted, and stored in a repository, to be used as input of Data Analytics (DA) algorithms.

\subsection{Metro-Haul Infrastructure}

The Metro-Haul infrastructure (spanning multiple geographic locations) relies on macroscopic nodes, combining networking, processing and storage resources. Such modular elements are composed of different components operating at different layers and technologies, and of different vendors, realizing hardware and software disaggregation inside the node. In particular, it implements layer 0-1 (optical domain), layer 2 transmission and switching (frame domain) and compute resources provided by local pool of servers to instantiate virtual network functions (VNFs) with configurable amount of processing, memory and storage. Concrete specializations of the generic architecture are at the Access Metro Edge nodes (AMEN nodes) to interface with heterogeneous access technologies and at the Metro Core Edge nodes (MCEN nodes): 1) AMEN nodes implement local/regional edge computing, aggregation and optical metro communication, along with access networks gateway, with both 5Gbased wireless and optical capabilities (e.g., fronthaul interfaces, CPRI, xPON) and 2) MCEN nodes are gateways towards the core transport network and comprise core-oriented capabilities.

(C) 2018 IEEE. Personal use of this material is permitted. Permission from IEEE must be obtained for all other uses, in any current or future media, including reprinting/republishing this material for advertising or promotional purposes,creating new collective works, for resale or redistribution to servers or lists, or reuse of any copyrighted component of this work in other works. DOI: 10.1109/ICTON.2018.8473832 


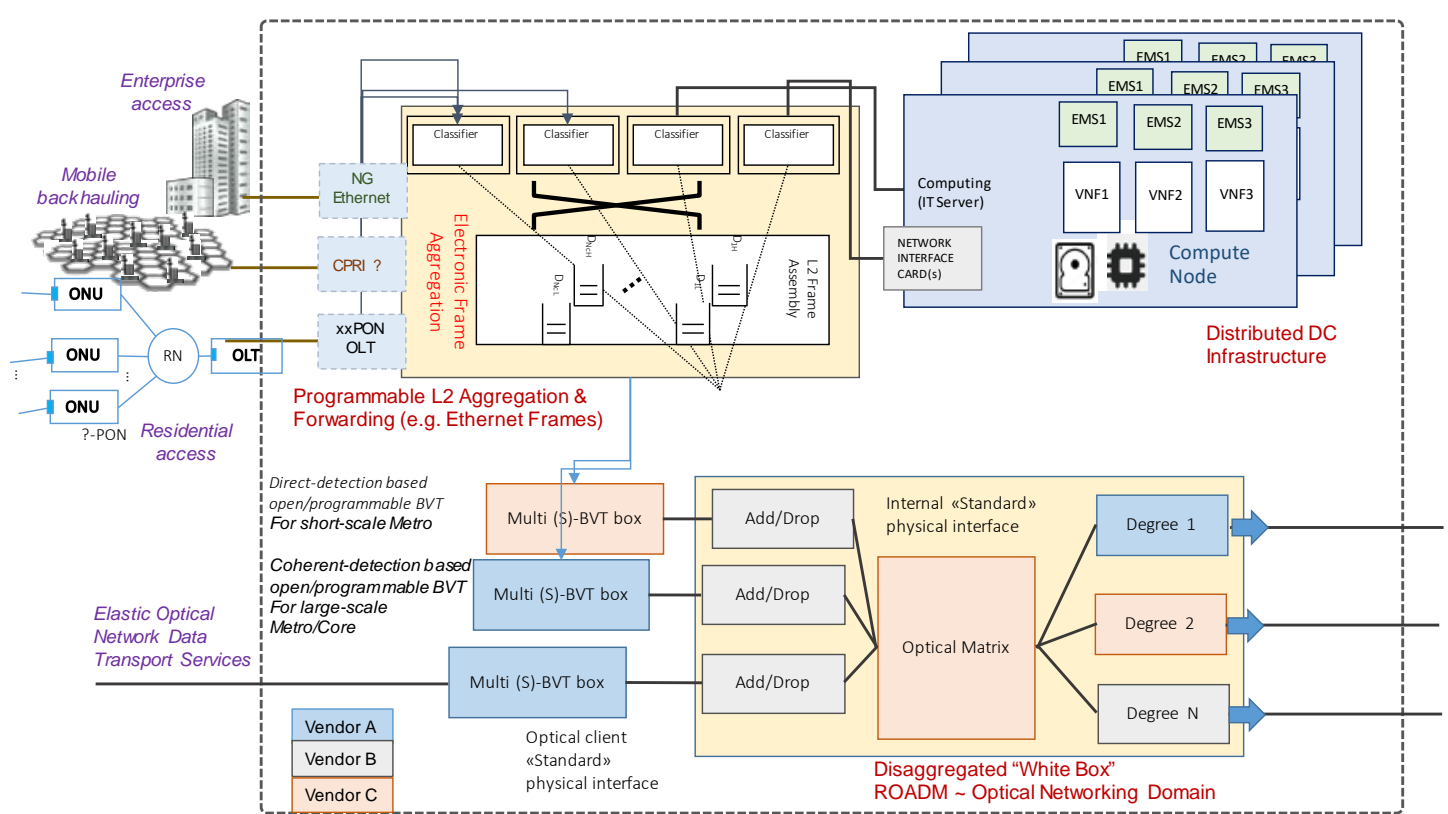

Figure 1 Macroscopic architecture of a Metro-Haul node, including Layer 2 framing and aggregation, IT resources and disaggregated optical network elements.

\subsection{Targeted Metro-Haul Services}

From a control and management point of view, we have identified a set of basic services to be developed. Our main targets are: 1) Network connectivity services. The core services considered include connectivity provisioning between MAC endpoints, IP addresses or optical layer ports; 2) Virtual Services. Metro-Haul include cloud resources to support the instantiation of VMs and VNFs, being able to configure VMs and attach virtual NICs to soft switches, 3) ETSI / Network Function Virtualization (NFV) Network Services and slicing. A key service offered is network slices. Within the scope of Metro-Haul, the main focus of a network slice is the ETSI NFV Network Service, a set of interconnected VNFs across the Metro-Haul infrastructure and 4) Monitoring Services. Cognitive network architectures have been proven to self-adapt the network in a costeffective manner (autonomic networking). Specifically, by applying data analytics to monitored data, control loops can be enabled, where analysis outcomes can be used to recommend network reconfiguration actions to the SDN controller. To dynamically deploy such services, we adopt SDN/NFV principles and frameworks, as we detail in Section 3, extended with additional dedicated systems. A monitoring and data analytics system is required to enable autonomic networking, i.e., to implement control loops that entail monitoring and reconfiguration or re-tuning. A dedicated network planning system is required to leverage existing network planning and algorithms that are able to perform function placement in a multi-layer context.

\section{USE CASES AND REQUIREMENTS FOR MONITORING AND DATA ANALYTICS}

In this section, we briefly mention some of the targeted use cases, in addition to the common failure detection and localization [7] and virtual network topology (VNT) adaptability based on daily traffic models [8].

Open Line Systems (OLS) monitoring. An OLS considers the disaggregation of a network into functional blocks (such as transponder, ROADMs and/or degrees in a switching fabric). Such disaggregation benefits from monitoring e.g. for alien wavelengths, and to optimize the power levels. Similarly, for coherent transmission, additional parameters (dispersion, differential group delay, BER) can be monitored and estimated, enabling closed loop control and optimization of bandwidth-variable transponders.

Transport Network Re-optimization. The collection of statistics and historical data regarding service requests allows to predict demands (e.g. using ML) and to re-optimize periodically ensuring an efficient network-resource optimization [5], towards an autonomic infrastructure that proactively self-configures and self-tunes may e.g. detect BER degradations, so notification can be sent toward the SDN controller with a recommended action to restore connections affected by the failure. Similarly, VNT are used in multi-layer networks and their computation is dependent on the input traffic matrices both at the packet and optical layer. Variations in traffic are difficult to accommodate in static or overprovisioned scenarios. Consequently, traffic matrices may be estimated periodically with dedicated passive and active probes, and algorithms may re-arrange connections.

VNF placement across transport networks. 5G services requiring the deployment of virtualized network functions (VNFs) may be constrained in terms of latency, jitter or bandwidth, especially if deployed in a metropolitan or WAN network. For example, some of the VNFs may be located near the end users, while those requiring additional computing power only in dedicated data centers. The joint network orchestration may rely 
on an external planner, optimizing provisioning while implementing medium and long term joint capacity planning of the IT and transport network resources, jointly addressing the dimensioning of the DCs and the dimensioning of the transport network, requiring active monitoring of heterogeneous resources.

\subsection{Requirements for Control interfaces}

Efficient Monitoring and Data Collection interfaces: adaptive network operation requires the development and adoption of advanced interfaces for information collection. Such interfaces need to overcome the limitations of existing ones to maximize efficiency and minimize latency, bandwidth usage, and data processing requirements. Additional requirements include, for example: i) to monitor the status of hundreds or thousands of entities or network elements typical of a large scale network; ii) to configure programmable pipe-lines in terms of asynchronous events and flexible filters for flexible data collection and notification, including expressive and domain embedded languages in a publisher-subscriber pattern; iii) the automatic discovery and monitoring of key but heterogeneous parameters. Existing approaches in network management are either too restrictive, often too slow or based on a "pull model", which requires additional processing to periodically poll the network elements, and limits scaling in large environments. Other monitoring methods may not accurate enough to detect issues caused by short-lived events or extremely bursty traffic.

Ongoing IETF drafts are being defined to enable notifications, e.g. by using the mechanisms allowing, notably, a network operator to: i) subscribe notifications on a per client basis, ii) configure what parameters to apply filtering and selective collection at the point of origin of the notification and iii) to request whether notifications are periodic, or event-driven, etc. The design framework required for streaming telemetry is different from traditional monitoring mechanisms, protocol requirements would include:1) Streaming of data in real-time, as fast as possible; 2) Supporting a push model; 3) Provide full access to operational data and 4) Support a policy model for defining time periods for collecting data, including tunable parameters (scale up or down). The development of SDN and telemetry (frameworks, protocols, and data models) is relatively recent, and based on three main principles: 1) a data modeling language which provides structured data models for technology and function specific data points, 2) a management protocol for encoding and carrying the data model information and 3) an operational process governing how the protocol interface is used and connections are managed. Practical examples for network telemetry solutions include, for example, the OpenConfig (http:// www.openconfig.net) project using YANG for data modeling, protobuff for encoding, gRPC for protocol transport and gRPC Network Operations Interface (gNOI) for operations. Open issues do exist and must be addressed to fulfil network telemetry objectives: a lack of dedicated telemetry protocol for data collection; data format consistency issues where different syntax and semantics may be used to retrieve the same type of data from different devices; data correlation so that collected information becomes meaningful.

Interfaces between components: by not having a monolithic design for the whole COM system, adopting a more micro-services architecture and decoupling the SDN controller, MDA and NP systems, we require interfaces between such systems. Macroscopically, we adopt REST interfaces and notifications. For example, the interface between the MDA and the SDN controller needs to support not only retrieving and synchronizing the contents of the operational SDN databases but also support bidirectional notifications on resource changes. In addition, notifications might include recommended actions. Likewise, the NP system requires access to the operational databases, monitoring information and to enable placement and computation. Different workflows are possible and being analysed.

\section{METRO-HAUL SERVICE PLATFORM}

NFVO Orchestrator and Hierarchical SDN Control. The NFV architecture defines the NFV Infrastructure (NFVI) deployed across multiple points of presence (NFVI-PoP) for supporting the instantiation of VMs, along with the Management and Orchestration (MANO) subsystem, which deals with the orchestration of VNFs and how to deploy them as components of the so called Network Services. OSM as the NFVO performs Service Orchestration, the part of service instantiation involving the functional split of the service into/amongst different VNFs and their logical interconnection (called VNF forwarding graphs) and Resource Orchestration that deals with the allocation of resources to support the VNFs and the logical links. Hierarchical SDN controllers are deployed for network orchestration.

Monitoring and Data Analytics subsystem: Monitored and telemetry data exported from the nodes is collected at the subsystem and stored into a distributed big data repository. This big data repository requires scalability and high availability without compromising performance. In the context of Metro-Haul, Apache Cassandra has been selected because of its capacity to effectively manage massive amounts of data. To enable centralized data analytics and KDD implementation in the domain controller, a KDD module is defined; it contains KDD processes that execute intensive data analytics tasks, either locally or delegated to a computing cluster. The Apache Spark framework will be considered as it is a fast and general engine for large-scale data processing. Regarding protocols, IPFIX for monitoring and gRPC for telemetry have been selected. 
Network Planning The aim is to optimize the resource allocation. The traffic generated by end-user oriented services have different requirements in terms of bandwidth, delay and QoS. For this reason, the intelligent optimization algorithms of the optimization module will apply different policies and strategies and will need data coming from the data plane. Open-source Net2Plan tool (www.net2plan.com) has been selected as the framework since it already models Networks Services, and service chains, easing algorithm development and testing for the new challenges coming from the transport \& IT joint network management. Net2Plan will be extended to interface with OSM, the SDN controllers and potentially with monitoring tools to keep a live network view, and provide allocation decisions.

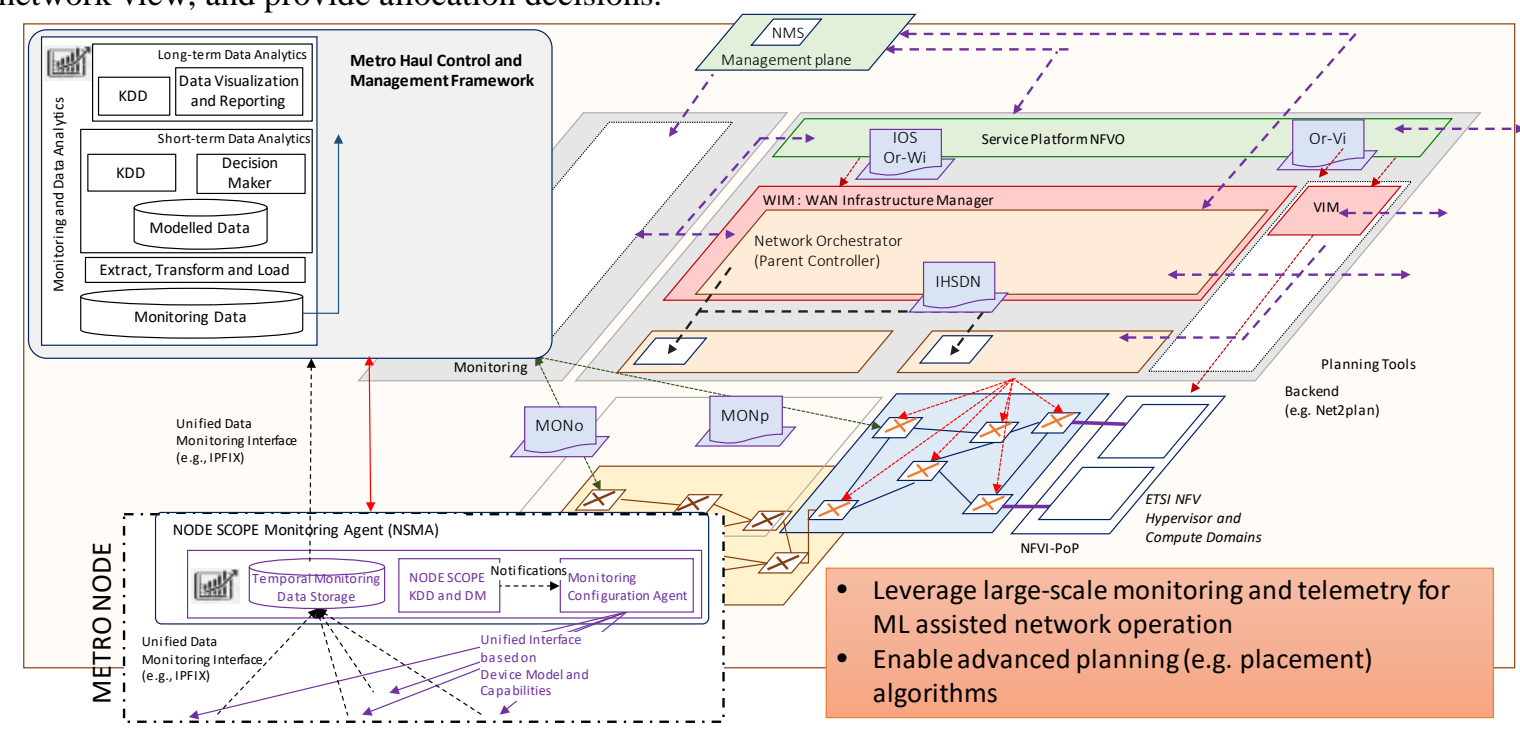

Figure 2 Metro-Haul Service Platform: NFV MANO system, a hierarchical packet over optical SDN control extended by dedicated Monitoring and Data Analytics Subsystem (MDA) and a Network Planner (NP) backend.

\section{CONCLUSIONS}

The deployment of 5G services is enabled by a control, orchestration and management architecture designed to cover metropolitan networks, conceived to interface $5 \mathrm{G}$ access networks with elastic core optical networks at multi $\mathrm{Tb} / \mathrm{s}$. The composing infrastructure nodes encompass networking, storage and processing resources, turn interconnected by open and disaggregated optical networks. Justified by use cases, the SDN based control is extended by subsystems like the Monitoring and Data Analytics or the in-operation planning backend.

\section{ACKNOWLEDGEMENTS}

The work presented in this paper has been partially funded by Spanish MINECO DESTELLO (TEC2015-69256R) project, and the European Commission through the H2020-ICT2016-2 METRO-HAUL (G.A.: 761727).

\section{REFERENCES}

[1] M. Bjorklung, ed. "Yang - A Data Modeling Language for the Network Configuration Protocol”, IETF Internet RFC 6020, October 2010

[2] A. Clemm, E. Voit, A. González Prieto, E. Nilsen-Nygaard, A. Bierman, B. Lengyel, "Subscribing to YANG datastore push updates”, draft-ietf-netconf-yang-push-05, February 28, 2017. Accessed: https://datatracker.ietf.org/doc/draft-ietfnetconf-yang-push/

[3] A. Clemm, A. González Prieto, E. Nilsen-Nygaard, A. Triàthy, S. Chisholm, H. Trevino, "Subscribing to Event Notifications", draft-ietf-netconf-rfc5277bis-01, October 27, 2016. Accessed: https://tools.ietf.org/html/draft-ietfnetconf-rfc5277bis-01

[4] Open Networking Foundation (ONF), "SDN Architecture”, Issue 1.1., 2016 ONF TR-521, available https://www.opennetworking.org/images/stories/downloads/sdn-resources/technical-reports/TR521_SDN_Architecture_issue_1.1.pdf

[5] L. Velasco, D. King, O. Gerstel, R. Casellas, A. Castro, V. López, “In-Operation network planning”, IEEE Commun. Mag, 52(1), 2014.

[6] ETSI "Network Functions Virtualisation (NFV); Management and Orchestration”, ETSI GS NFV-MAN 001 V1.1.1 (2014-12), available: http://www.etsi.org/deliver/etsi_gs/NFV-MAN/001_099/001/01.01.01_60/gs_NFVMAN001v010101p.pdf

[7] A. P. Vela et al., "Soft Failure Localization during Commissioning Testing and Lightpath Operation [Invited]," IEEE/OSA J. of Optical Communications and Networking (JOCN), vol. 10, pp. A27-A36, 2018.

[8] F. Morales et al., "Dynamic Core VNT Adaptability based on Predictive Metro-Flow Traffic Models,” IEEE/OSA J. of Optical Communications and Networking (JOCN), vol. 9, pp. 1202-1211, 2017. 